\title{
HYDRODYNAMIC INERTIA COEFFICIENTS FOR A SLENDER BODY WITH A SAIL*
}

\author{
$\mathrm{Br}$ \\ THEODORE R. GOODMAN AND RALPH GREIF** \\ Allied Research Associates, Inc., Boston Massachusetts
}

Introduction. In the course of studying the hydrodynamics of a submerged submarine it becomes necessary to calculate the hydrodynamic inertia coefficients of a body whose cross-section consists of a circle with one sail (fin). The simpler inertia coefficients for this shape appear in the open literature, although somewhat obscured by the presence of what, for this problem, would be considered extraneous fins. The inertia coefficient which gives rise to rolling moment due to roll velocity is not in the literature, and it is principally with the calculation of this coefficient that the present paper is concerned.

Analysis. To determine the forces and moments on a rigid slender body moving through an infinite incompressible fluid where the motion of the fluid is irrotational and at rest far from the body, requires the evaluation of the six components of the symmetric inertia tensor of the cross section of the body

$$
m_{i k}=m_{k i}=-\rho \oint \phi_{i} \frac{\partial \phi_{k}}{\partial n} d s \quad j, k=1,2,3 .
$$

Refer to Lamb [1], Munk [2], Jones [3], Spreiter [4] and Bryson [5].

The cross section of the configuration is mapped conformally onto a circle in order to determine the velocity potentials for the low-speed two-dimensional motion of the cross section. The inertia coefficients may then be determined from Eq. (1).

Consider a slender body of revolution of radius $a\left(x_{3}\right)$, bearing a sail of height $t\left(x_{3}\right)-a\left(x_{3}\right)$, as shown in cross section in Fig. 1. Because of the plane of symmetry of the cross section, we see that:

$$
\text { a) } m_{23}=0 .
$$

That is, a motion in the $x_{2}$ direction cannot produce a rolling moment. In addition,

$$
\text { b) } m_{12}=0 \text {. }
$$

That is, a motion in the $x_{1}$ direction cannot produce a force in the $x_{2}$ direction.

The sail does not affect the inertia coefficient $m_{22}$ since it lies along the streamlines of the flow. Therefore, the case of no sail suffices to obtain $m_{22}$. The result is

$$
\frac{m_{22}}{\rho \pi a^{2}}=1
$$

\footnotetext{
${ }^{*}$ Received Oct. 20, 1960. This work was performed for the Office of Naval Research under Contract Nonr-2343(00).

${ }^{* *}$ Graduate student at Harvard University.
} 
The expression for $m_{11}$ may be taken directly from Summers [6] or Bryson [7]. The result is

$$
\frac{m_{11}}{\rho \pi a^{2}}=\left\{1+\frac{a^{2}}{4 t^{2}}\left(\frac{t}{a}-1\right)^{2}\left[\left(\frac{t}{a}\right)^{2}+6\left(\frac{t}{a}\right)+1\right]\right\} .
$$

Equation (5) has been plotted in Fig. 2.
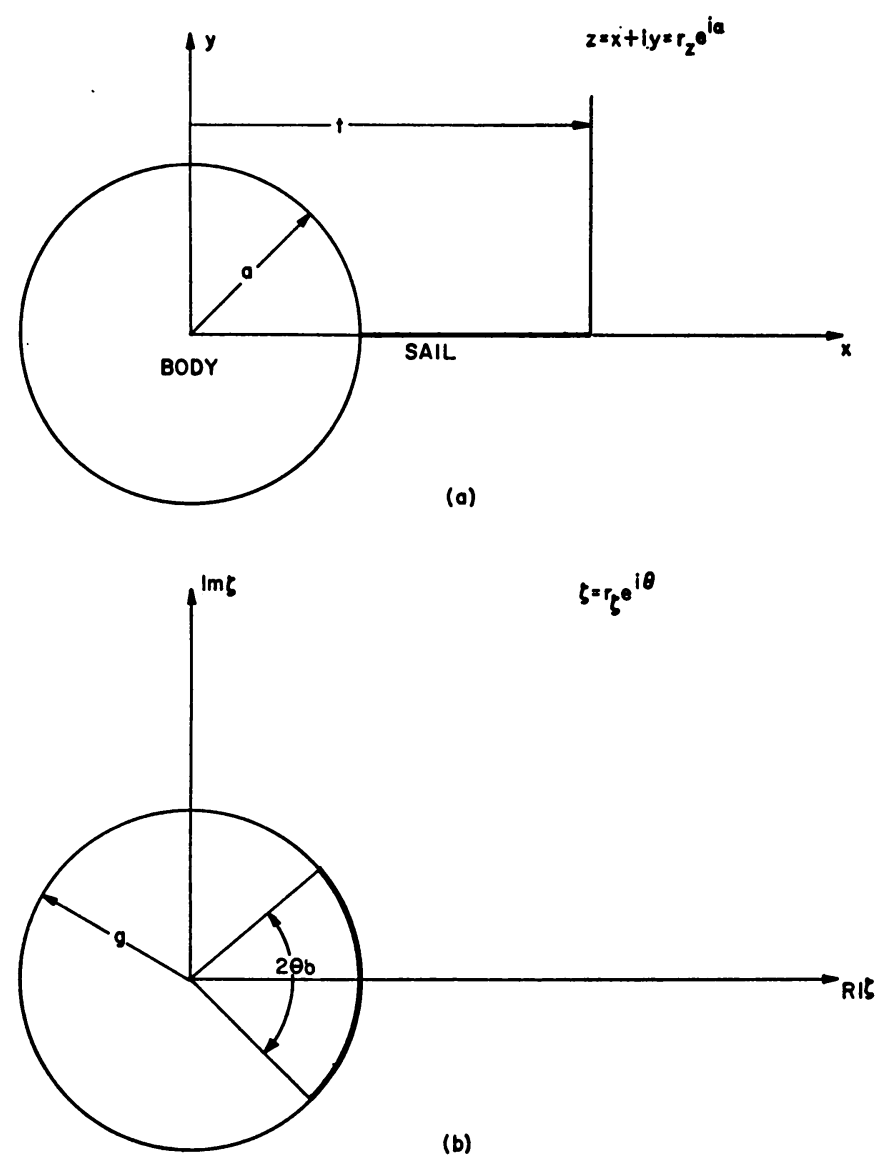

FIG. 1. Mapping of a circle with a sail onto a circle (Eq. 9)

The expression for $m_{13}$ may be taken directly from Bryson [7]. The result is* $\frac{m_{13}}{\rho \pi a^{3}}=\frac{(t / a-1)^{2}}{\pi}\left[\frac{\left(2 \beta_{0}+\pi\right)}{16} \frac{(t / a+1)^{4}}{(t / a)^{3}}+\frac{(t / a-1)}{4(t / a)^{1 / 2}}\left(1+\frac{14}{3} \frac{1}{t / a}+\frac{1}{(t / a)^{2}}\right)\right]$,

where

$$
\beta_{0}=\frac{\theta_{0}}{2}=\sin ^{-1}\left(\frac{t / a-1}{t / a+1}\right) .
$$

Equation (6) has also been plotted in Fig. 2.

*An error in sign has been corrected which makes $m_{13}$ positive. 


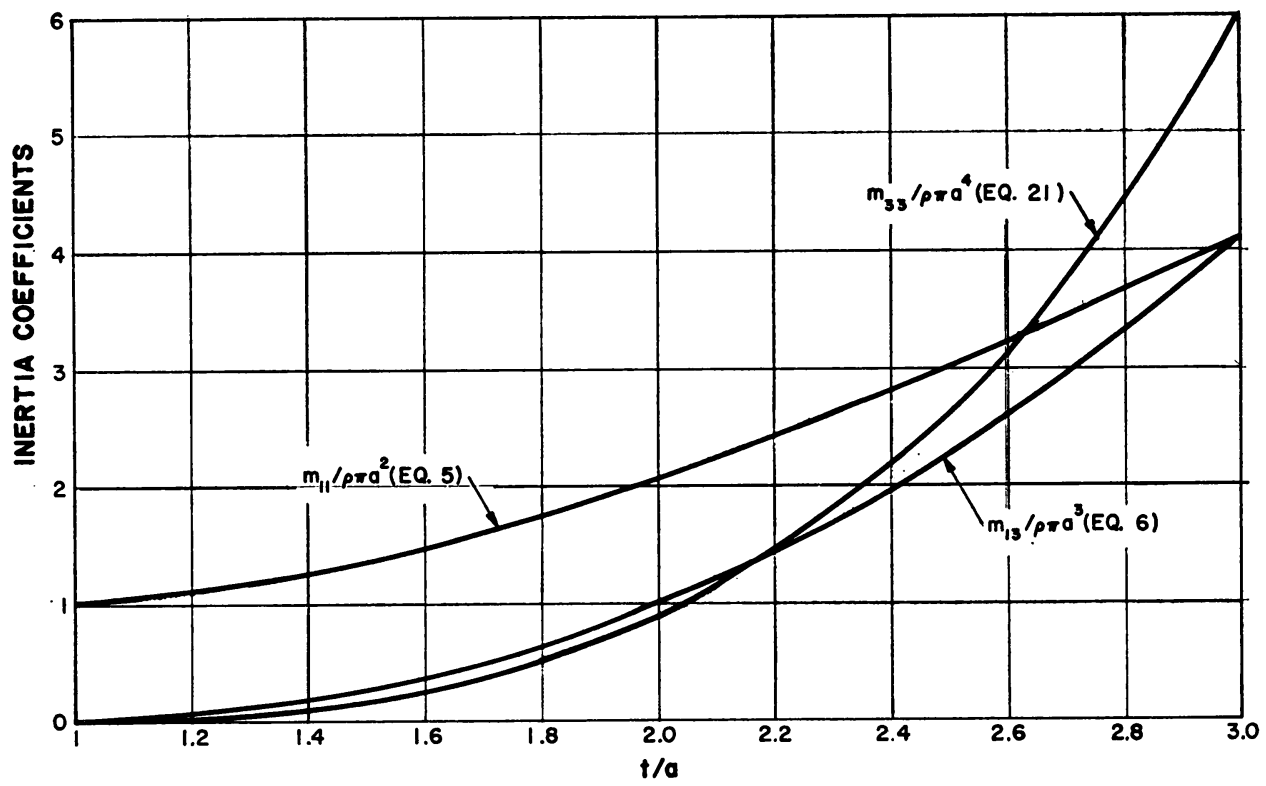

FIG. 2. Inertia coefficients as functions of sail length-radius ratio

The apparent additional moment of inertia, $m_{33}$, for a body of revolution with a sail is not given in the literature and will now be obtained.

It is required to find a solution, $\phi_{3}(x, y)$, to the two-dimensional Laplace equation in the $z=x+i y$ plane satisfying the boundary conditions

on the sail

on the body

$$
\left.\frac{\partial \phi_{3}}{\partial y}(x, y)\right|_{y=0}=x \quad a<x<t,
$$

$$
\left.\frac{\partial \phi_{3}}{\partial r_{z}}\left(r_{z} \cos \alpha, r_{z} \sin \alpha\right)\right|_{r_{z}=a}=0,
$$

where $(x, y)$ are a set of right handed, cartesian coordinates and the longitudinal dimension is measured positive downstream from the body nose. Note that we have assumed unit angular velocity about this axis.

The conformal transformation

where

$$
z\left(1+\frac{a}{z}\right)^{2}-2 g=\zeta+\frac{g^{2}}{\zeta}
$$

$$
g=\frac{t}{4}\left(1+\frac{a}{t}\right)^{2}
$$

maps the cross section of the body in the $z(=x+i y)$ plane (Fig. 1) on the circle $|\zeta|=g$ in the $\zeta$ plane, the sail appearing as an arc extending from $-\theta_{0}$ to $\theta_{0}$, where

$$
\cos \theta_{0}=\frac{2 a}{g}-1=\frac{6 a-t-\left(a^{2} / t\right)}{2 a+t+\left(a^{2} / t\right)} .
$$


On the sail the mapping function given by Eq. (9) and (10) reduces to

$$
x=[g(1+\cos \theta)-a]+\{g(1+\cos \theta) \cdot[g(1+\cos \theta)-2 a]\}^{1 / 2} .
$$

The boundary conditions (7) and (8) transform to

$$
\begin{aligned}
& \left.\frac{\partial \phi_{3}}{\partial r_{\zeta}}\left(r_{\zeta}, \theta\right)\right|_{r_{\zeta}=g}=\frac{2 x \sin \theta}{1-\left(a^{2} / x^{2}\right)} \quad-\theta_{0}<\theta<\theta_{0}, \\
& \left.\frac{\partial \phi_{3}}{\partial r_{\zeta}}\left(r_{\zeta}, \theta\right)\right|_{r_{\zeta}=g}=0 \quad|\theta|>\theta_{0} .
\end{aligned}
$$

The problem has thus been reduced to an exterior Neumann problem for a circle and the solution for $\phi_{3}$ is given by

$$
\phi_{3}\left(r_{\zeta}, \theta\right)=\left.\frac{g}{2 \pi} \int_{0}^{2 \pi} \frac{\partial \phi}{\partial r_{\zeta}}\right|_{r_{\zeta}=g} \ln \left|1-\frac{2 g}{r_{\zeta}} \cos (\theta-\psi)+\left(\frac{g}{r_{\zeta}}\right)^{2}\right| d \psi .
$$

On the circle $\zeta=g e^{i \theta}$ the solution for the potential $\phi_{3}$ is given by

$$
\phi_{3}(g, \theta)=\left.\frac{g}{\pi} \int_{0}^{2 \pi} \frac{\partial \phi}{\partial r_{\zeta}}\right|_{r_{\zeta}=0} \ln \left|2 \sin \frac{\theta-\psi}{2}\right| d \psi .
$$

All integrals are to be evaluated in the Cauchy sense. Equations (13) and (14) are substituted into (16), and $x$ is eliminated by virtue of Eq. (12). Then, after an integration by parts to eliminate the logarithm there is obtained:

$$
\begin{aligned}
\phi_{3}(g, \theta)=-\frac{g^{2}}{\pi} \sin \theta \int_{0}^{\theta_{0}} & \left\{\cos \frac{\psi}{2}\left(2 \cos ^{2} \frac{\psi}{2}-\cos ^{2} \frac{\theta_{0}}{2}\right)\left(\cos ^{2} \frac{\psi}{2}-\cos ^{2} \frac{\theta_{0}}{2}\right)^{1 / 2}\right. \\
+ & \left.2 \cos ^{2} \frac{\psi}{2}\left(\cos ^{2} \frac{\psi}{2}-\cos ^{2} \frac{\theta_{0}}{2}\right)\right\}\left(\cos ^{2} \frac{\psi}{2}-\cos ^{2} \frac{\theta}{2}\right)^{-1} d \psi,
\end{aligned}
$$

where $\cos \theta_{0}$ is defined in Eq. (11). Carrying out the integration it is found that

$$
\begin{aligned}
\phi_{3}(g, \theta)=-\frac{g^{2}}{\pi} \sin \theta\left\{\sin \theta_{0}+\left(\theta_{0}+\pi\right)\left(1-\cos \theta_{0}+\cos \theta\right)\right. & \\
& \left.+\frac{\left(\cos \theta-\cos \theta_{0}\right)(1+\cos \theta)}{\sin \theta} \ln \left|\frac{\tan \frac{g_{0}}{2}+\tan \frac{\theta}{2}}{\tan \frac{\theta_{0}}{2}-\tan \frac{\theta}{2}}\right|\right\} .
\end{aligned}
$$

Now, from Eq. (1)

$$
m_{33}=-\rho \oint \phi_{3} \frac{\partial \phi_{3}}{\partial n} d s
$$

and on the body surface

$$
\frac{\partial \phi_{3}}{\partial n} d s=-x d x-y d y .
$$


But Eq. (20) equals zero on the body surface; $|z|=a$; hence, the integration need only be confined to the sail. The resulting integral is quite tedious. The result is

$$
\begin{aligned}
\frac{m_{33}}{\pi \rho a^{4}}= & \frac{4}{\pi^{2} \eta^{4}}\left\{\beta_{0}\left(\pi+\beta_{0}\right)\left(\frac{9}{2}-8 \eta+4 \eta^{2}\right)\right. \\
& +\beta_{0}(9-10 \eta)[\eta(1-\eta)]^{1 / 2}+\frac{\pi}{2}\left(14-25 \eta+15 \eta^{2}-5 \eta^{3}\right)[\eta(1-\eta)]^{1 / 2} \\
& \left.+(1-\eta)\left[\frac{\pi^{2}}{8}\left(9-7 \eta+\eta^{2}+\eta^{3}\right)+\eta\left(\frac{9}{2}-2 \eta-2 \eta^{2}\right)\right]\right\},
\end{aligned}
$$

where

$$
\eta=\frac{a}{g}=\frac{4 t / a}{\left(1+\frac{t}{a}\right)^{2}}=\cos ^{2} \beta_{0}
$$

Equation (21) has been plotted in Fig. 2.

As a check on this result, it will be noted that the value of $m_{33}$ is given by Miles for the case of a body of revolution bearing two fins of equal length which are mounted 180 degrees apart [8]. For very small fins, the value of $m_{33}$ given by Eq. (21) for the single-finned body should be one-half of the value given by Miles for the two-finned body, since, if the fins are small, they will not mutually interfere. Carrying out the limiting process for both cases bears out this relationship. An additional check may be made by proceeding to the limit as the body shrinks to zero. This case is given by Bryson [9], and it can be shown that Eq. (21) yields the correct limit.*

\section{REFERENCES}

1. H. Lamb, Hydrodynamics, 6th ed., Dover, New York, 1932, Chap. VI

2. M. M. Munk, The aerodynamic forces on airship hulls, N. A. C. A. Report No. 184, 1924

3. R. T. Jones, Properties of low aspect ratio pointed wings at speeds below and above the speed of sound, N. A. C. A. Report No. 835,1946

4. J. R. Spreiter, The aerodynamic forces on slender plane and cruciform wing and body combinations, N. A. C. A. Report No. 962,1950

5. A. E. Bryson, Stability derivatives for a slender missile with application to a wing-body-vertical-tail configuration, J. Aeronaut. Sci. 20, No. 5, 297 (May 1953)

6. R. G. Summers, On determining the additional apparent mass of a wing-body-vertical-tail cross section, Readers' Forum, J. Aeronaut. Sci. 20, No. 12, 856 (Dec. 1953)

7. A. E. Bryson, Comment on the stability derivatives of a wing-body-vertical-tail configuration, Readers' Forum, J. Aeronaut. Sci. 21, No. 1, 59 (Jan. 1954)

8. J. W. Miles, The potential theory of unsteady supersonic flow, Cambridge University Press, 1959, p. 171

9. A. E. Bryson, Evaluation of the inertia coefficients of the cross section of a slender body, Reader's Forum, J. Aeronaut. Sci. 21, No. 6, 424 (June 1954)

*The authors are indebted to the referee for pointing out this limiting case. 